\title{
Clinical Analysis of Sputum Gram Stains and Cultures to Improve the Quality of Sputum Cultures
}

\author{
Dong-Hyun Lee ${ }^{1}$ and Sunjoo Kim ${ }^{2}$ \\ ${ }^{1}$ Department of Laboratory Medicine, Gyeongsang National University Hospital, Jinju; 2Department of Laboratory Medicine, \\ Gyeongsang National University Changwon Hospital, Changwon, Korea
}

Corresponding author:

Sunjoo Kim

Department of Laboratory Medicine, Gyeongsang National University Changwon Hospital, 11 Samjeongja-ro, Seongsan-gu, Changwon 51472, Korea Tel +82-55-214-3072

Fax +82-55-214-3087

E-mail sjkim8239@hanmail.net

Received: November 3, 2019

Revised: December 21, 2019

Accepted: January 6, 2020

This is an Open Access article distributed under the terms of the Creative Commons Attribution Non-Commercial License (http://creativecommons.org/licenses/ by-nc/4.0) which permits unrestricted non-commercial use, distribution, and reproduction in any medium, provided the original work is properly cited.
Background: Sputum Gram stains and cultures are standard tests for the diagnosis of lower respiratory tract infections. Analysis reports of the sputum quality are scarce in relation to cultures as well as clinical diagnosis.

Methods: A total of 1,523 sputum specimens, requested for culture, were evaluated for their quality using the modified Murray-Washington (MW) grouping system in association with the culture results. The clinical diagnosis was investigated for the culture positive cases.

Results: There was a significant difference in bacterial growth (odds ratio, 2.01; $P=0.0164$ ) and diagnosis of pneumonia (odds ratio, 4.18; $P=0.002$ ) between the acceptable groups (groups 4-5) and the unacceptable groups (groups 1-3). However, one-quarter did not belong to the current MW group. More than half of the sputum cultures were in group 6, with the lowest positive rate $(18.0 \%)$. Overall, $10 \%$ of the sputum cultures were related to a clinical diagnosis of pneumonia.

Conclusions: The MW grouping system should be revised, because onequarter did not belong to the current group. Further evaluation of group 6 will be needed. Improvement of sputum cultures is required to enhance the diagnosis of pneumonia.

\section{(Lab Med Qual Assur 2020;42:33-39)}

Key Words Pneumonia, Culture, Streptococcus pneumoniae, Quality improvement

\section{INTRODUCTION}

Lower respiratory tract infections including communityacquired pneumonia (CAP) or hospital-acquired pneumonia (HAP) are major public health concerns, causing a variety of complications and high mortality [1-3]. More than 1.5 million adults are estimated to be hospitalized due to CAP, with an estimated 100,000 deaths annually in the United States [4]. Chest X-ray is the primary test for the diagnosis of lower respiratory tract infections $[5,6]$. Sputum culture is also a confirmative test to isolate the causative organisms of lower respiratory tract infections, such as CAP, bronchiolitis, and bronchitis, and therefore, is routinely performed in clinical microbiological laboratories [5,7]. Sputum Gram staining is associated with sputum culture results [7-10], and highquality sputum provides useful information or guides the monotherapy $[1,5,11,12]$. However, the clinical usefulness 
of sputum cultures and Gram stains is questioned due to possible contamination of oropharyngeal normal flora and the complexity of etiological pathogens $[6,10]$. Adequate collection of sputum must be accomplished to obtain proper test results. Deeply expectorated sputum could serve as a high-quality specimen. Rinsing the mouth with water and coughing deeply early in the morning is recommended [8]. However, it is not easy to obtain a highquality specimen due to the lack of proper education or poor patient conditions. Some reports demonstrated that up to one-third of the patients with bacterial pneumonia were unable to produce sputum $[13,14]$.

Several criteria have been proposed to evaluate the quality of sputum samples. These criteria have different combinations and cutoffs for the minimum number of squamous epithelial cells (SEC) and/or polymorphonuclear leukocytes (PMN) per low-power field (LPF; $100 \times$ objective) [15-17], but none of these guidelines are considered superior. Currently, the modified MurrayWashington (MW) grouping system is primarily used to evaluate the quality of sputum in Korea [17]. This grouping system consists of six groups according to the numbers of SEC and PMN per LPF. There is a consensus that groups 1-3 are unacceptable, whereas groups 4 and 5 are acceptable. However, controversy persists regarding the acceptability of group 6 , which shows less than 10 SEC and PMN each, per LPF. In the original MW grouping system, group 6 ( $<10 \mathrm{SEC}$ ) was categorized as acceptable [17]. Bartlett [16] also used SEC and PMN to score sputum quality. Although these grouping systems have been used for several decades, their clinical utility has not been well established. An in-depth analysis of culture results and clinical data is needed in relation to the classification of the sputum groups. Additionally, we found that some samples do not belong to groups 1-6, and no research has been undertaken, to date, for the interpretation of these unclassified groups. Concomitant blood culture is recommended to increase the chance of isolating causative CAP organisms in addition to sputum cultures $[5,6]$. However, some guidelines recommend only blood cultures instead of sputum cultures for the diagnosis of CAP [6].
We evaluated the clinical relevance of sputum cultures using the MW grouping system and examined the frequency of bacterial growth in each group. We also analyzed the results of requested concomitant blood cultures.

\section{MATERIALS AND METHODS}

\section{Study population}

From December 2016 to February 2017, a total of 1,523 consecutive sputum specimens, requested for cultures and Gram stains, were included from Gyeongsang National University Hospital. Electronic medical records were reviewed to analyze the requested concomitant blood cultures, presence of pneumonia, prior antibiotic use before sputum culture, and antibiotic change after receiving the sputum culture results from the patients with positive cultures. The clinical diagnosis was based on clinical signs and symptoms of lower respiratory tract infections and the presence of a new infiltrate, on chest radiographs, that was at least segmental and not due to any preexisting conditions or other known causes.

This study was approved by the institutional review board of Gyeongsang National University Hospital (IRB approval no., GNUH 2019-10-027-002).

\section{Microbiological laboratory tests}

Gram stain smears were prepared from the most visually purulent portion of each sputum specimen. The sputum quality was assessed microscopically and each sputum sample was grouped, as outlined by the MW grouping system [17]. The most purulent portion of each specimen was inoculated onto blood, chocolate, and MacConkey's agars. Then samples were streaked out by a medical technician using a standard 4-quadrant streaking method and incubated at $35^{\circ} \mathrm{C}$ with $5 \% \mathrm{CO}_{2}$ for 48 hours. Agar plates were examined at 16-24 hours and 48 hours, and the predominant organisms were identified and quantified, by an experienced technician, according to the furthest quadrant with visible colonies. Respiratory potential pathogens included Staphylococcus aureus, Streptococcus pyogenes, Streptococcus dysgalactiae, 
Streptococcus agalactiae, Streptococcus pneumoniae, Nocardia spp., Moraxella catarrhalis, Haemophilus influenzae, Haemophilus parainfluenzae, and Gramnegative bacilli $[1,11]$. Normal throat flora, such as $\alpha^{-}$ or $\Upsilon$-streptococcus, coagulase-negative staphylococcus, Micrococcus spp., Neisseria spp., Corynebacterium spp., were discarded. Blood cultures were performed using the BacT/Alert 3D system (bioMérieux, Marcy-l'Etoile, France) with FA plus (aerobic) and FN plus (anaerobic) bottles. An amount of 5-10 mL was inoculated into each bottle. Two sets were produced for each episode. Biochemical identification and antibiotic susceptibility tests were conducted using the Vitek-2 system (bioMérieux). To standardize reporting across each technician, uniform standard operating procedures, on-site training, and internal and external quality controls were established.

\section{Statistical analysis}

Frequencies are expressed as a percentage (\%) of the categorical variables. We compared the results for bacterial growth and pneumonia between the groups using the chi-square test and Fisher's exact test. All of the statistical analyses were performed using SAS ver. 9.4 (SAS Institute Inc., Cary, NC, USA). P-values $<0.05$ were considered statistically significant.

\section{RESULTS}

\section{Patient demographics and sputum characteristics}

A total of 1,523 sputum specimens were requested for culture during the study period. The ratio of males to females was 1.6:1 (Table 1). The percentages of expectorated sputum, suction sputum, transtracheal aspirates, and bronchial aspirates were $87.0 \%, 8.3 \%, 3.8 \%$, and $0.9 \%$, respectively. Respiratory potential pathogens were identified in $22.6 \%$ of the samples. A total of $87.8 \%$ of sputum culture positive cases $(n=344)$ were concurrently requested with the blood cultures. The blood cultures (37.2\%) were requested from the emergency department among the sputum culture positive cases, indicating that the majority of the specimens came from in-patients. The positive rate of blood cultures was $8.9 \%$, and $6.6 \%$
Table 1. Characteristics of the study population, types of sputum, concomitant blood cultures, and antibiotic usage

\begin{tabular}{|c|c|}
\hline Characteristic & Value \\
\hline Median age (yr) & 70 \\
\hline Male:female & $1.6: 1$ \\
\hline No. of sputum specimens & 1,523 \\
\hline \multicolumn{2}{|l|}{ Specimen types } \\
\hline Expectorated sputum & $1,325(87.0)$ \\
\hline Suction sputum & $127(8.3)$ \\
\hline Transtracheal aspirates & $58(3.8)$ \\
\hline Bronchial aspirates & $13(0.9)$ \\
\hline No. of bacterial identifications & $344(22.6)$ \\
\hline No. of co-ordered blood cultures & $302(87.8)$ \\
\hline $\begin{array}{l}\text { Positive blood cultures in positive sputum } \\
\text { culture cases }\end{array}$ & $27(8.9)$ \\
\hline The same species as sputum culture & $8(2.3)$ \\
\hline Different species from sputum culture & $19(6.6)$ \\
\hline \multicolumn{2}{|l|}{$\begin{array}{l}\text { Antibiotic use in positive sputum culture } \\
\text { patients }\end{array}$} \\
\hline $\begin{array}{l}\text { Prior antibiotic usage before sputum } \\
\text { culture }\end{array}$ & $97(28.2)$ \\
\hline $\begin{array}{l}\text { Antibiotic change after confirmation of } \\
\text { sputum culture result }\end{array}$ & $79(23.0)$ \\
\hline
\end{tabular}

Values are presented as number or number (\%).

of the blood cultures yielded different species from the sputum cultures. Prior antibiotic usage before sputum culture accounted for $28.2 \%$, and antibiotic changes after receiving the sputum culture results were observed in $23.0 \%$ of the sputum culture positive patients.

\section{Murray-Washington grouping system}

The proportion of sputum samples belonging to MW groups 1 to 6 were $2.9 \%, 2.0 \%, 6.2 \%, 3.5 \%, 1.0 \%$, and $56.9 \%$, respectively, among the 1,523 samples (Fig. 1).

Respiratory potential pathogens grew in $31.8 \%(14 / 44)$, 23.3\% (7/30), 33.7\% (32/95), 44.4\% (24/54), 60.0\% (9/15), and $18.0 \%$ (156/867), respectively, for each group (Table 2). The proportion of specimens that did not belong to any of the MW groups was $27.5 \%(418 / 1,523)$, and bacteria grew in $24.4 \%(102 / 418)$ in this unclassified group. The group of SEC $10-25, \mathrm{PMN}<10$ was the most prevalent among the unclassified groups. The growth of 
respiratory potential pathogens was significantly higher in the acceptable groups compared to unacceptable groups (odds ratio [OR], 2.01; 95\% confidence interval [Cl], 1.133.56; $P=0.0164)$. In addition, the diagnosis of pneumonia was significantly higher in the acceptable groups (OR, 4.18; $95 \% \mathrm{Cl}, 1.64-10.60 ; P=0.002$ ).

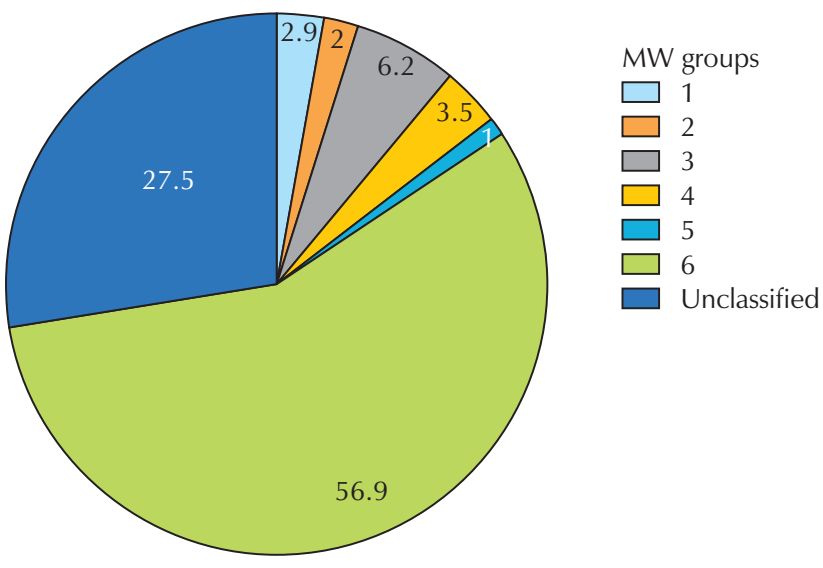

Fig. 1. Distribution (\%) of MW groups $(n=1,523)$. Unclassified groups which is not included in groups 1-6 accounted for $27.5 \%$. Abbreviation: MW, Murray and Washington.
The unclassified group showed no significant differences in the growth of potential respiratory pathogens $(P=$ 0.0833 ) but showed significant differences in the diagnosis of pneumonia compared to the unacceptable groups $(P=0.0168)$. There was no significant difference in etiological pathogens, such as gram-positive, gram-

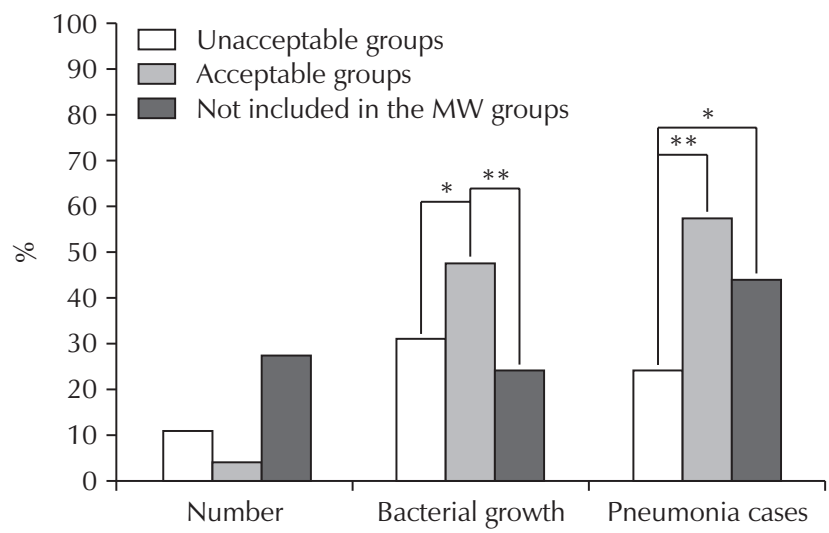

Fig. 2. Comparison of bacterial growth rates and diagnosis of pneumonia among the unacceptable (groups 1-3), acceptable (groups 4-5), and not included in the MW groups. Abbreviation: MW, Murray and Washington. ${ }^{\star} P<0.05,{ }^{\star \star} P<0.01$.

Table 2. Growth of respiratory potential pathogens and clinical diagnosis of pneumonia according to MW groups and the among unclassified groups using the MW grouping system

\begin{tabular}{|c|c|c|c|c|c|}
\hline & No. (\%) & $\begin{array}{c}\text { Bacterial } \\
\text { growth (\%) }\end{array}$ & $P$-value & $\underset{(\%)^{*}}{\text { Pneumonia }}$ & $P$-value ${ }^{+}$ \\
\hline Total & 1,523 & 344 & & 150 & \\
\hline MW grouping system & 1,105 & 242 & & 105 & \\
\hline Group 1 & $44(2.9)$ & $14(31.8)$ & $<0.001$ & $1(7.1)$ & 0.006 \\
\hline Group 2 & $30(2.0)$ & $7(23.3)$ & & $1(14.3)$ & \\
\hline Group 3 & $95(6.2)$ & $32(33.7)$ & & $11(34.4)$ & \\
\hline Group 4 & $54(3.5)$ & $24(44.4)$ & & $15(62.5)$ & \\
\hline Group 5 & $15(1.0)$ & $9(60.0)$ & & $4(44.4)$ & \\
\hline Group 6 & $867(56.9)$ & $156(18.0)$ & & $73(46.8)$ & \\
\hline Not included in the MW grouping system & $418(27.5)$ & $102(24.4)$ & 0.0833 & $45(44.1)$ & 0.0168 \\
\hline SEC $10-25$, PMN $<10$ & $182(12.0)$ & $34(18.7)$ & & $11(32.4)$ & \\
\hline SEC 10-25, PMN 10-25 & $78(5.1)$ & $24(30.8)$ & & $10(41.7)$ & \\
\hline SEC $<10$, PMN $10-25$ & $158(10.4)$ & $44(27.8)$ & & $24(54.5)$ & \\
\hline
\end{tabular}

Abbreviations: MW, Murray and Washington; SEC, squamous epithelial cells; PMN, polymorphonuclear leukocytes.

${ }^{*}$ The percentage of pneumonia cases was calculated by dividing with number of bacterial growth positive patients. ${ }^{\dagger}$ The $P$-value was calculated between each MW group and not included groups compared with the acceptable groups (groups 4-5). 
negative, and fungi, according to the MW groups (data not presented) (Fig. 2).

\section{DISCUSSION}

Clinical decisions for antibiotic treatment of lower respiratory infections are aided by the results of Gram stains and sputum cultures $[1,9,11]$. However, sputum Gram stains are sometimes unreliable and ambiguous $[8,18]$. Therefore, the optimal collection of high-quality sputum specimens and the correct isolation and identification of colonies are important $[8,10]$. However, obtaining an acceptable specimen is sometimes difficult because of the possibility of contamination from normal oropharyngeal flora [7]. In addition, microbiological expertise is required for the appropriate selection of the colonies of potential respiratory pathogens. Sputum cultures are rather subjective and difficult to standardize technically $[10,18]$. The yield of sputum cultures was $22.6 \%$ in this study. Among these potential respiratory pathogens ( $\mathrm{N}=344), 43.6 \%$ were associated with pneumonia. Therefore, overall $10 \%$ of the sputum specimens were related to the clinical diagnosis of pneumonia.

Expectorated sputum is the most common lower respiratory tract specimen collected by microbiology laboratories. Expectorated sputum is often difficult to obtain as many specimens are mixed with upper airway specimens. In our study, the unacceptable groups (1-3) accounted for $11.1 \%$, whereas the acceptable groups (4-5) accounted for only $4.5 \%$. In contrast, group 6 was predominant (56.9\%), and a surprisingly large proportion (27.5\%) did not belong to any of the groups in the current MW grouping system (Fig. 1).

In other studies, the percentage of acceptable quality specimen ranged from $40 \%-70 \%$, as compared to $4.5 \%$ in our study. However, these studies differed from ours with respect to the subjects, who were patients with pneumonia [9,12], and the criteria for a good sample, which was "SEC <10/LPF" [19].

The bacterial growth rate was highest in the acceptable groups $4-5(47.8 \%)$ and lowest in group $6(18.0 \%)$
$(P<0.001)$. However, pneumonia diagnosis in acceptable groups $(46.8 \%)$ was significantly higher than that in unacceptable groups (24.5\%) ( $P=0.004)$. Group 6 fared worse than the acceptable group in terms of bacteria growth detection but better in terms of pneumonia diagnosis. Therefore, further research on group 6 is needed, and new interpretation of the MW grouping system is necessary.

Blood culture was established as a major part of the investigation of CAP [20]. However, the necessity of mandatory blood cultures remains controversial for the diagnosis of CAP [21]. More evidence-based studies are needed to elucidate the advantage of routine blood cultures for the diagnosis of CAP or HAP. The positive rate of blood cultures, among the patients who had potential respiratory pathogens, was $8.9 \%$, and a large portion had a different pathogen from the sputum culture in our study.

There are several limitations to this study. This is a retrospective study rather than a prospective study. The medical records were reviewed only for the patients showing positive results in their sputum cultures, and not for all the patients. There was no differentiation between CAP and HAP. Mortality, immunocompromised patients such as those suffering from neutropenia, prior other medical treatments, or clinical severity were not analyzed. One-quarter of the subjects received antibiotics before sputum collection, which might have affected the sputum culture results. Active antibiotic stewardship was not applied at our institute, thus lowering the change of antibiotics even after receiving the antibiotic susceptibility test results.

In conclusion, approximately $10 \%$ of the sputum cultures were related to the clinical diagnosis of pneumonia. The lower yield might have been due to the inappropriate requests for sputum cultures or prior antibiotic usage. One-quarter did not belong to the current MW groups. More than half of the sputum samples were in group 6 and showed the lowest positive rate. More large-scale studies will be needed on MW group 6. Improvement of sputum culture quality is required to enhance the diagnosis of pneumonia. The beneficial effects of additional 
blood cultures should be carefully reassessed and further

infections.

studied, for the diagnosis of lower respiratory tract

\section{REFERENCES}

1. Musher DM, Thorner AR. Community-acquired pneumonia. N Engl J Med 2014;371:1619-28.

2. Chalmers J, Campling J, Ellsbury G, Hawkey PM, Madhava H, Slack M. Community-acquired pneumonia in the United Kingdom: a call to action. Pneumonia (Nathan) 2017;9:15.

3. Giuliano KK, Baker D, Quinn B. The epidemiology of nonventilator hospital-acquired pneumonia in the United States. Am J Infect Control 2018;46:322-7.

4. Ramirez JA, Wiemken TL, Peyrani P, Arnold FW, Kelley R, Mattingly WA, et al. Adults hospitalized with pneumonia in the United States: incidence, epidemiology, and mortality. Clin Infect Dis 2017;65:1806-12.

5. Baron EJ, Miller JM, Weinstein MP, Richter SS, Gilligan PH, Thomson RB Jr, et al. A guide to utilization of the microbiology laboratory for diagnosis of infectious diseases: 2013 recommendations by the Infectious Diseases Society of America (IDSA) and the American Society for Microbiology (ASM)(a). Clin Infect Dis 2013;57:e22-e121.

6. Niederman MS, Mandell LA, Anzueto A, Bass JB, Broughton WA, Campbell GD, et al. Guidelines for the management of adults with community-acquired pneumonia: diagnosis, assessment of severity, antimicrobial therapy, and prevention. Am J Respir Crit Care Med 2001;163:1730-54.

7. Garcia-Vazquez E, Marcos MA, Mensa J, de Roux A, Puig J, Font C, et al. Assessment of the usefulness of sputum culture for diagnosis of community-acquired pneumonia using the PORT predictive scoring system. Arch Intern Med 2004;164:1807-11.

8. Flournoy DJ. Microbiology: interpreting the sputum Gram stain report. Lab Med 1998;29:763-8.

9. Miyashita N, Shimizu H, Ouchi K, Kawasaki K, Kawai Y, Obase Y, et al. Assessment of the usefulness of sputum Gram stain and culture for diagnosis of community-acquired pneumonia requiring hospitalization. Med Sci Monit 2008;14:CR171-6.

10. Campbell S, Forbes BA. The clinical microbiology laboratory in the diagnosis of lower respiratory tract infections. J Clin Microbiol 2011;49(9 Suppl):S30-3.

11. Gleckman R, DeVita J, Hibert D, Pelletier C, Martin R. Sputum gram stain assessment in community-acquired bacteremic pneumonia. J Clin Microbiol 1988;26:846-9.

12. Roson B, Carratala J, Verdaguer R, Dorca J, Manresa F, Gudiol F. Prospective study of the usefulness of sputum Gram stain in the initial approach to community-acquired pneumonia requiring hospitalization. Clin Infect Dis 2000;31:86974.

13. Bartlett JG, Mundy LM. Community-acquired pneumonia. N Engl J Med 1995;333:1618-24.

14. Marrie TJ. Community-acquired pneumonia. Clin Infect Dis 1994;18:501-13.

15. Wong LK, Barry AL, Horgan SM. Comparison of six different criteria for judging the acceptability of sputum specimens. J Clin Microbiol 1982;16:627-31.

16. Bartlett RC. Medical microbiology: quality cost and clinical relevance. New York (NY): John Wiley \& Sons, 1974.

17. Murray PR, Washington JA. Microscopic and baceriologic analysis of expectorated sputum. Mayo Clin Proc 1975;50:339-44.

18. Nagendra S, Bourbeau P, Brecher S, Dunne M, LaRocco M, Doern G. Sampling variability in the microbiological evaluation of expectorated sputa and endotracheal aspirates. J Clin Microbiol 2001;39:2344-7.

19. Murdoch DR, Morpeth SC, Hammitt LL, Driscoll AJ, Watson NL, Baggett HC, et al. The diagnostic utility of induced 
sputum microscopy and culture in childhood pneumonia. Clin Infect Dis 2017;64(suppl_3):S280-8.

20. Waterer GW, Wunderink RG. The influence of the severity of community-acquired pneumonia on the usefulness of blood cultures. Respir Med 2001;95:78-82.

21. Chalasani NP, Valdecanas MA, Gopal AK, McGowan JE Jr, Jurado RL. Clinical utility of blood cultures in adult patients with community-acquired pneumonia without defined underlying risks. Chest 1995;108:932-6. 